\title{
Translating neurochemistry: benefit of joint behavioral and in vivo imaging studies in the rat for neuropsychiatric disorders
}

\author{
Nikolaus $\mathrm{S}^{*}$, Beu M, Antke C and Mueller HW \\ Clinic of Nuclear Medicine, University Hospital Duesseldorf, Heinrich-Heine University, Moorenstr. 5, 40225 Duesseldorf, Germany
}

Anxiety disorder (AD), major depressive disorder (MDD) and schizophrenia (SZ) share the characteristic of imbalanced $\gamma$-amino butyric acid (GABA), dopamine (DA) and serotonin (5-HT) function. The individual disorders, however, greatly differ as to the affected brain regions, the affected synaptic constituents and the direction of dysfunction in terms of either sensitization or desensitization of receptor and transporter binding sites [1-3]. While, in $\mathrm{AD}, \mathrm{GABA}_{\mathrm{A}}$ receptor $(\mathrm{R})$ desensitization involves the whole nigrostriatal and mesolimbocortical system, in SZ, GABA $\mathrm{R}$ dysfunction is confined to frontal and temporal cortex and, in MDD, even appears to be normal. In contrast, in $\mathrm{AD}$, inhibitory $\mathrm{D}_{2}$ Rs are merely desensitized in the dorsal striatum, while, in SZ, they are additionally dysfunctional in thalamus, temporal cortex and midbrain. In MDD, no $\mathrm{D}_{2} \mathrm{R}$ desensitization is evident - rather, $\mathrm{D}_{2} \mathrm{Rs}$ are sensitized in the cingulate. Excitatory $\mathrm{D}_{1} \mathrm{R}$ binding sites in both $\mathrm{AD}$ and MDD are desensitized in the dorsal striatum, whereas in, in SZ, their sensitization, besides the ventral striatum, involves the entire neocortex. Dopamine transporter (DAT) function is most widely inpaired in MDD with reductions in substantia nigra, cingulate, amygdala and prefrontal cortex, whereas, in SZ, DAT desensitization is confined to the dorsal striatum and, in $\mathrm{AD}$, no DAT dysfunction is evident at all. The most extensive impairment of inhibitory $5-\mathrm{HT}_{1} \mathrm{R}$ binding is observed in $\mathrm{AD}$ with 5 - $\mathrm{HT}_{1} \mathrm{R}$ desensitization in the midbrain and throughout limbic system as well as neocortex, whereas, in $\mathrm{SZ}, 5-\mathrm{HT}_{1} \mathrm{R}$ desensitization is confined to midbrain, amygdala and occipitotemporal regions. In MDD, 5- $\mathrm{HT}_{1} \mathrm{R}$ are also desensitized in the midbrain, but sensitized in the limbic parahippocampal gyrus. The most widely spread dysfunction of excitatory $5-\mathrm{HT}_{2} \mathrm{Rs}$ is observed in MDD, with a sensitization of $5-\mathrm{HT}_{2} \mathrm{Rs}$ in the thalamus and a desensitization throughout both limbic system and neocortex. In contrast, in $\mathrm{AD}, 5-\mathrm{HT}_{2} \mathrm{Rs}$ are sensitized in the temporal cortex and desensitized in the ventral striatum, while, in $\mathrm{SZ}, 5-\mathrm{HT}_{2} \mathrm{R}$ desensitization involves the entire neocortex. In $\mathrm{AD}$, the serotonin transporter (SERT) is desensitized in thalamus, hippocampus and amygdala, while, in MDD, SERT desensitization is even more extensive, involving - apart from thalamus and amygdala - also midbrain, dorsal striatum and prefrontal cortex. In addition, in MDD, SERT is sensitized in the insula. Interestingly, no alterations of SERT are evident in SZ.

Symptoms of $\mathrm{AD}, \mathrm{MDD}$ and $\mathrm{SZ}$ range from long-lasting, intense anxiety, obsessive thoughts, irrational compulsive behaviors, anhedonia, severely depressed mood, feelings of excessive guilt, delusions and hallucinations to disorganized speech and movement disturbances. The observed patterns of regional receptor and transporter sensitizations and/or desensitizations indicate that the emotional and behavioral changes characteristic for the individual disorders may be related to regional neurochemical alterations of receptor and transporter function. There is little knowledge, however, as to the precise link between a specific aberration of receptor or transporter function and a given neuropsychiatric symptom.

Behavioral studies in small animal lesion models have taught us much about the relation between functions such as "mood", "memory" or "locomotion" to specific brain regions. Moreover, the combination of behavioral and in vivo microdialysis studies have related specific functions to regional neurotransmitter levels. Labelling of microscopic slices with suitable (including radioactive) compounds has revealed the distributions of receptors and transporters in the brain, while tract-tracing and patch-clamp electrophysiological studies have helped to develop network models for a multitude of behavioral functions. The next important step will be to translate alterations of receptor or transporter binding into alterations of emotional, cognitive or motor behaviors.

A recent study of motor and exploratory behaviors and $\mathrm{D}_{2} \mathrm{R}$ binding in the rat after pre-treatment with L-DOPA has shown that lower $\mathrm{D}_{2} \mathrm{R}$ binding in the dorsal striatum (reflecting an increase of synaptic DA) was associated with a decrease in exploratory activity and grooming, but an increase in sitting frequency [4]. Moreover, pre-treatment with the $G A B A_{A} R$ agonist muscimol reduced $D_{2} R$ binding in dorsal striatum, ventral striatum, thalamus, substania nigra/ventral tegmental area and hippocampus. Thereby, lower $\mathrm{D}_{2} \mathrm{R}$ binding was associated with a reduction of motor/exploratory activity (Figure 1) and an elevation of grooming [5]. In contrast, pre-treatment with the $\mathrm{GABA}_{\mathrm{A}} \mathrm{R}$ antagonist bicuculline elevated $\mathrm{D}_{2} \mathrm{R}$ binding in dorsal striatum and thalamus. Also here, lower $\mathrm{D}_{2} \mathrm{R}$ binding could be related to lower motor/exploratory activity but an increase of sitting duration [5]. These studies after pharmacological challenges in the rat constitute an important step towards unravelling the complex interdependencies of behaviors and the neurochemistry of both DA as well as GABA.

In order to extend our knowledge on the exact association between a given behavioral parameter and the regulation state of specific binding sites in various brain region, future investigations must be directed towards jointly assessing receptor/transporter binding and emotional, cognitive or motor behaviors in the same rat or mouse with

${ }^{\star}$ Correspondence to: Susanne Nikolaus, Clinic of Nuclear Medicine, University Hospital Duesseldorf, Heinrich-Heine University, Moorenstr. 5, 40225 Duesseldorf, Germany, E-mail: susanne.nikolaus@uni-duesseldorf.de

Received: June 28, 2018; Accepted: July 04, 2018; Published: July 06, 2018 


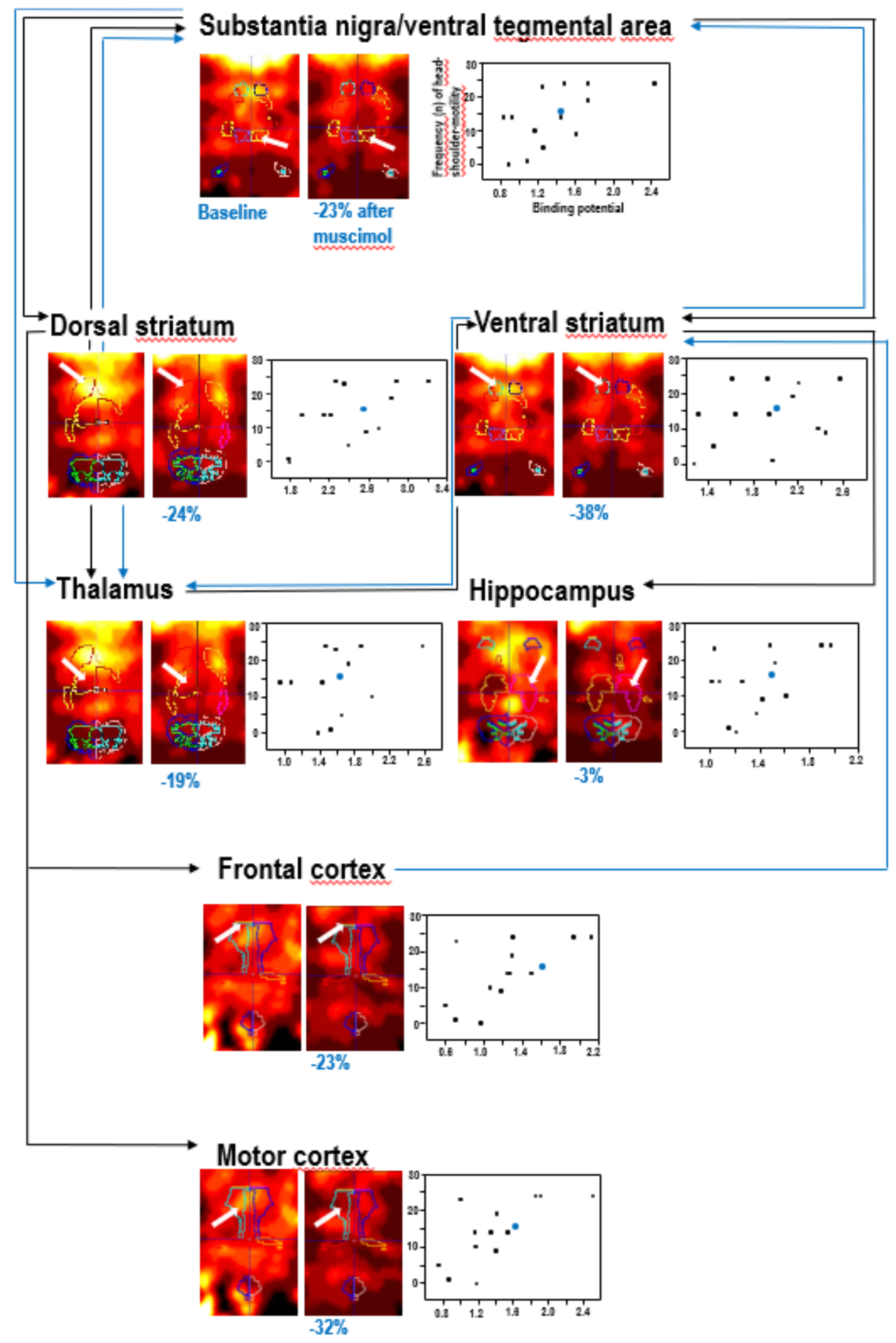

Figure 1. An example of joint assessment of $\mathrm{D}_{2} \mathrm{R}$ binding (using [123 I] iodobenzamide and small animal SPECT) in baseline and after challenge with muscimol (1 mg/kg i.p.) and of exploratory behavior after muscimol challenge in the rat. The depicted exploratory behavior is the frequency of head-shoulder motility during the last 5 minutes of the testing time. The figures shows radioligand accumulations in substantia nigra/ventral tegmental area, dorsal striatum, ventral striatum, thalamus, hippocampus, frontal cortex and motor cortex on coronal slices in baseline and post-challenge in the same rat. For each region, the percentual difference of $\mathrm{D}_{2} \mathrm{R}$ binding after muscimol relative to baseline in this specific animal is given below the post-challenge image. The scatter plots show the relation between the binding potentials ( $\mathrm{x}$-axis) and the frequencies of the head-shoulder motility in minute 25 -30 post challenge ( $\mathrm{y}$-axis) for each region. The circles represent the individual rats, which underwent joint investigation of in vivo $\mathrm{D}_{2} \mathrm{R}$ binding and behavioral parameters (in total, $\mathrm{n}=16$ ). In those animals, muscimol induced significant reductions of $\mathrm{D}_{2} \mathrm{R}$ binding in substantia nigra/ventral tegmental area, dorsal striatum, ventral striatum, thalamus and hippocampus. The values of the rat, whose regional $\mathrm{D}_{2} \mathrm{R}$ images are presented left to the scatter plots, are highlighted in blue. Substantia nigra/ventral tegmental area, dorsal striatum, ventral striatum, thalamus, hippocampus, frontal cortex and motor cortex are key regions of the nigostriatal and mesolimbocortical system. Charted are DAergic (black) and GABAergic (blue) pathways between these regions. In vivo imaging and behavioral data have been previously published (Nikolaus et al., Front Behav Neurosci 12: article 38). Also for the summary of the anatomic connections the reader is referred to this paper 
suitable paradigms. Examples are elevated plus maze, radial-arm maze or open field for the assessment of fear-related behaviors, cognitive skills and motor/exploratory behaviors, respectively. Promising tools, in this respect, may be devices such as the RatCAP [6], a dedicated small animal PET, which enables even simultaneous performance of in vivo imaging and behavioral studies.

Since regional increases or decreases of receptor/transporter binding permit to gauge alterations of synaptic neurotransmitter levels, the joint assessment of behaviors and regional DA, 5-HT, GABA, glutamate or acetylcholine binding sites will help to establish comprehensive models for individual behavioral parameters in terms of related presynaptic transporter function, pre-/postsynaptic receptor function and synaptic neurotransmitter levels. This holds for the regulatory interactions not only between individual brain regions but also between neurotransmitter systems. If such investigations are complemented by joint studies of receptor/transporter binding and emotional, cognitive or motor parameters in suitable rat or mouse models of $\mathrm{AD}, \mathrm{MDD}$ and SZ (genetic or otherwise, such as infliction of inescapable severe stressors, social isolation or prepulse inhibition of startle), functional networks can be compared between healthy and impaired animals. Ultimately, patterns of neurochemical aberrations will arise, which may be translated into the behavioral operationalizations of specific symptoms. This is essential for understanding the exact impact(s) of disease-specific alterations of transporter and receptor binding observed in human in vivo imaging studies (see above) on the expression of a given neuropsychiatric phenotype.

\section{References}

1. Nikolaus S, Hautzel H, Mueller HW (2014) Focus on GABA receptor function - a comparative analysis of in vivo imaging studies on neuropsychiatric disorders. Nuklearmedizin 53: 227-37.

2. Nikolaus S, Mueller HW, Hautzel H (2016) Different patterns of 5-HT receptor and transporter dysfunction in neuropsychiatric disorders - a comparative analysis of in vivo imaging findings. Rev Neurosci 27: 27-59.

3. Nikolaus S, Mamlins E, Hautzel H, Mueller HW (2018) Acute anxiety disorder, major depressive disorder, bipolar disorder and schizophrenia are related to different patterns of nigrostriatal and mesolimbic dopamine dysfunction. Rev Neurosci in press.

4. Nikolaus S, Beu M, De Souza Silva AM, Huston JP, Hautzel H, Mattern C, Antke C, Mueller HW (2016) Relationship between L-DOPA-induced reduction in motor and exploratory activity and striatal dopamine $\mathrm{D}_{2}$ receptor binding in the rat. Front Behav Neurosci 9: article 352 .

5. Nikolaus S, Wittsack J, Beu M, De Souza Silva MA, Huston JP, Mueller-Lutz A Wickrath F, Antke C, Antoch G, Mueller HW, Hautzel H (2018) GABAergic control of nigrostriatal and mesolimbic dopamine in the rat brain. Front Behav Neurosci 12: article 38 .

6. Schulz D, Vaska P (2011) Integrating PET with behavioral neuroscience using RatCAP tomography. Rev Neurosci 22: 647-55.

Copyright: (C)2018 Nikolaus S. This is an open-access article distributed under the terms of the Creative Commons Attribution License, which permits unrestricted use, distribution, and reproduction in any medium, provided the original author and source are credited. 